\title{
Three novel mutations in the carnitine-acylcarnitine translocase (CACT) gene in patients with CACT deficiency and in healthy individuals
}

\author{
Takao Fukushima ${ }^{1}$, Hidetoshi Kaneoka ${ }^{1,2}$, Tetsuhiko Yasuno ${ }^{1}$, Yukari Sasaguri ${ }^{2}$, Tomoko Tokuyasu ${ }^{2}$, \\ Kuniko Tokoro ${ }^{3}$, Toshiyuki Fukao ${ }^{4}$ and Takao Saito ${ }^{1,5}$
}

Carnitine-acylcarnitine translocase (CACT) and carnitine palmitoyltransferase II (CPT2) are key enzymes for transporting longchain fatty acids into mitochondria. Deficiencies of these enzymes, which are clinically characterized by life-threatening nonketotic hypoglycemia and rhabdomyolysis, cannot be distinguished by acylcarnitine analysis performed using tandem mass spectrometry. We had previously reported the CPT2 genetic structure and its role in CPT2 deficiency. Here, we analyzed the CACT gene in 2 patients diagnosed clinically with CACT deficiency, 18 patients with non-traumatic rhabdomyolysis and 58 healthy individuals, all of whom were confirmed to have normal CPT2 genotypes. To facilitate CACT genotyping, we used heatdenaturing high-performance liquid chromatography (DHPLC), which helped identify five distinct patterns. The abnormal heteroduplex fragments were subjected to CACT-specific DNA sequencing. We found that one patient with CACT deficiency, Case 1, carried c.576G $>A$ and c.199-10t $>$ g mutations, whereas Case 2 was heterozygous for c.106-2a $>t$ and c.576G $>A$. We also found that one patient with non-traumatic rhabdomyolysis and one healthy individual were heterozygous for $c .804 \mathrm{delG}$ and the synonymous mutation $\mathrm{c} .516 \mathrm{~T}>\mathrm{C}$, respectively. In summary, $\mathrm{c} .576 \mathrm{G}>\mathrm{A}, \mathrm{c} .106-2 \mathrm{a}>\mathrm{t}$ and $\mathrm{c} .516 \mathrm{~T}>\mathrm{C}$ are novel CACT gene mutations. Among the five mutations identified, three were responsible for CACT deficiency. We have also demonstrated the successful screening of $C A C T$ mutations by DHPLC.

Journal of Human Genetics (2013) 58, 788-793; doi:10.1038/jhg.2013.103; published online 3 October 2013

Keywords: $\beta$-oxidation; CACT deficiency; carnitine-acylcarnitine translocase (CACT); CPT2 deficiency; denaturing high-performance liquid chromatography (DHPLC); rhabdomyolysis

\section{INTRODUCTION}

Carnitine-acylcarnitine translocase (CACT), as well as carnitine palmitoyltransferase II (CPT2), has pivotal roles in the carnitine cycle, which is the $\beta$-oxidation pathway of long-chain fatty acids. ${ }^{1}$ The $C A C T$ gene is known to be associated with the SLC25 gene family, SLC25A20. In 1997, the human CACT cDNA was cloned and sequenced by Huizing et al. ${ }^{2}$ The entire gene, spanning about $42 \mathrm{~kb}$ on chromosome 3 p21.31, contains 9 exons and codes a 301-amino-acid protein. ${ }^{3,4}$ Cases of CACT deficiency are rare, and only 40 cases at most have been reported worldwide. ${ }^{5-7}$ Although defects in CACT induce lethal neonatal episodes of coma due to hypoketotic hypoglycemia, cardiomyopathy, cardiac arrhythmia and rhabdomyolysis, quite a few patients exhibit milder phenotypes compatible with longer life spans. ${ }^{8,9}$

CACT deficiency (OMIM No. 212138) cannot be easily differentiated from severe cases with CPT2 deficiency (OMIM No. 255110) on the basis of clinical manifestations and blood acylcarnitine profiles analyzed using tandem mass spectrometry; ${ }^{10-13}$ however, genetic analysis could be utilized to distinguish between the two conditions. CACT deficiency is so rare that the characteristics of the CACT gene are not yet fully understood. In this study, we analyzed the CACT gene in 2 patients diagnosed clinically with CACT deficiency, 18 Japanese patients with non-traumatic rhabdomyolysis and 58 healthy Japanese individuals. As little information is available regarding CACT polymorphisms and mutations, we introduced heat-denaturing high-performance liquid chromatography (DHPLC) to screen for the CACT gene. ${ }^{14}$ To confirm that DHPLC would be suitable for screening these gene mutations and variants, the following strategy was carried out. First, we sequenced all fragments showing heteroduplex patterns; second, we sequenced all the exons of the $C A C T$ gene in randomly selected individuals; third, we included data for two patients who had died of CACT deficiency; and fourth, the data were confirmed by family studies.

In this study, we identified mutations responsible for CACT deficiency and screened CACT mutations with DHPLC. To the best

${ }^{1}$ Division of Nephrology and Rheumatology, Department of Internal Medicine, Fukuoka University School of Medicine, Fukuoka, Japan; ${ }^{2}$ Division of Medical Sciences, Fukuoka University School of Nursing, Fukuoka, Japan; ${ }^{3}$ Department of Neonatal Medicine, Gifu Prefectural General Hospital, Gifu, Japan; ${ }^{4}$ Department of Pediatrics, Graduate School of Medicine, Gifu University, Gifu, Japan and ${ }^{5}$ General Medical Research Center, Fukuoka University School of Medicine, Fukuoka, Japan

Correspondence: Professor H Kaneoka, Division of Medical Sciences, Fukuoka University School of Nursing, 7-45-1 Nanakuma, Jonan-ku, Fukuoka 814-0180, Japan. E-mail: kaneokah@fukuoka-u.ac.jp

Received 2 April 2013; revised 4 September 2013; accepted 6 September 2013; published online 3 October 2013 
of our knowledge, the two infant cases are only the second and third cases wherein the genotypes of Japanese patients with CACT deficiency have been fully confirmed.

\section{SUBJECTS AND METHODS}

\section{Patients diagnosed clinically with CACT deficiency}

Case 1. The patient was the second child of non-consanguineous Japanese parents. His sister was in good health. His mother had pregnancy-induced hypertension. He was born at 36 weeks and 5 days of gestation by cesarean section. He had decreased respiratory functions and experienced asphyxia. At 2 days of age, repeated apnea, muscular hypotonia, irritability, oliguria, hypoglycemia, liver dysfunction and cryptogenic rhabdomyolysis were noted. Although CPT2 or CACT deficiency was suspected on the basis of acylcarnitine profiles of plantar blood spots (Table 1), the subsequent DNA analysis performed at our laboratory showed that his CPT2 gene was normal (data not shown). ${ }^{15}$ The patient was clinically diagnosed with CACT deficiency. At the age of 2 years and 9 months, he died of symptoms resembling those of Reye syndrome. (Case 1 is going to be reported in Japanese from a clinical point of view in an upcoming issue of the Journal of the Japan Pediatric Society by Sugiyama, et al.)

Case 2. The patient was the second child of non-consanguineous Japanese parents. His elder sister was healthy. The pregnancy was uneventful, and at 37 weeks of gestation, he was born by normal vaginal delivery. At 2 days of age, he suddenly became cyanotic and flaccid and then went into cardiac arrest. He died at 3 days of age. Tandem mass spectrometry analysis of acylcarnitines in a postmortem blood spot card showed increases in C14, C16 and C18 acylcarnitines (Table 1), which led us to consider a defect in either CACT or CPT2. After the patient died, the physician in charge brought the blood disc of the patient and whole blood from his parents to our laboratory for genetic analysis in order to reach a final diagnosis. Direct DNA sequencing performed on genomic DNA from the parents confirmed the absence of mutations in the CPT2 gene (data not shown). ${ }^{15}$ We highly suspected that the patient had died of CACT deficiency.

\section{Patients with non-traumatic rhabdomyolysis}

Eighteen patients with non-traumatic rhabdomyolysis (12 men and 6 women; mean age \pm s.d., $51.1 \pm 19.6$ years) were retrospectively recruited from the outpatient clinic of Fukuoka University Hospital and Clinic, Fukuoka, Japan. The mean \pm s.d. value of their peak serum creatine kinase levels was $22522.7 \pm 7224.4 \mathrm{IUl}^{-1}$. The patients had no history of statin therapy. DNA sequencing carried out at our laboratory indicated that all patients had normal CPT2 genotypes (data not shown). ${ }^{15}$

\section{Healthy volunteers}

The 58 individuals in our healthy panel included 47 men and 11 women (mean \pm s.d. age, $28.3 \pm 7.1$ years). They had no recent history of either heavy sport activities or specific drug usage. The CPT2 genotypes of these individuals were determined by DNA sequencing and were found to be normal without exception (data not shown)..$^{15}$

Table 1 Acylcarnitine levels in dried blood spots from Cases 1 and 2

\begin{tabular}{lccc}
\hline Acylcarnitine & Case 1 $(\mu \mathrm{m})$ & Case 2 $(\mu \mathrm{m})$ & Upper $95 \%$ limit $(\mu \mathrm{m})$ \\
\hline C0 & 9.37 & 11.85 & 60.0 \\
C14 & 0.66 & 1.35 & 0.4 \\
C14:1 & 0.17 & 0.56 & 0.1 \\
C14-OH & 0.064 & 0.24 & 0.050 \\
C16 & 4.77 & 12.07 & 1.9 \\
C16:1 & 0.78 & 1.84 & 0.3 \\
C18:1 & ND & 3.07 & 2.1 \\
\hline
\end{tabular}

Abbreviation: ND, not determined.

\section{Amplification of genomic DNA}

Genomic DNA purified from peripheral blood cells was PCR amplified using nine pairs of $C A C T$-specific primers (Table 2), as previously described. ${ }^{4,16} \mathrm{PCR}$ conditions were as follows: $95^{\circ} \mathrm{C}$ for $1 \mathrm{~min} ; 30$ cycles each of $95^{\circ} \mathrm{C}$ for $1 \mathrm{~min}$, specific annealing temperature for $1 \mathrm{~min}$, and $72^{\circ} \mathrm{C}$ for $1 \mathrm{~min}$, followed by a final extension step at $60^{\circ} \mathrm{C}$ for $10 \mathrm{~min}$

\section{DNA sequence analysis}

CACT-specific direct DNA sequencing was performed on all PCR products using the Big Dye Terminator v3.1 Cycle Sequencing Kit (Applied Biosystems, Foster City, CA, USA), and the results were analyzed on the automated ABI Prism 310 Genetic Analyzer (Applied Biosystems), as described by the manufacturer. Cumulative sequences were compared with the CACT sequence published in the OMIM database (http://www.ncbi.nlm.nih.gov/nuccore/ 319918843).

\section{Homoduplex or heteroduplex formation}

CACT-specific PCR products were purified using Microcon (Millipore Corp., Billerica, MA, USA). PCR product of each test was mixed with the comparable PCR product from a normal donor. The mixtures were denatured at $95^{\circ} \mathrm{C}$ for $10 \mathrm{~min}$ and then slowly cooled down to $65^{\circ} \mathrm{C}$ at a rate of $1{ }^{\circ} \mathrm{Cmin}-1$. Following 5-min incubation at $65^{\circ} \mathrm{C}$, the samples were cooled to $4{ }^{\circ} \mathrm{C}$ at a rate of $1{ }^{\circ} \mathrm{C}$ every $5 \mathrm{~s}$.

\section{DHPLC analysis}

PCR-amplified products were analyzed using the Nucleic Acid Fragment Analysis System (Transgenomic, Inc., San Jose, CA, USA). The results have been presented in the form of chromatographic peaks by using the Navigator software. Complete sequence-matched hybridization produced a single homoduplex peak, whereas hybridization with sequences containing mutation(s) resulted in heteroduplexes and aberrant peaks with different retention times. PCR products that showed heteroduplex patterns by DHPLC were subjected to $C A C T$-specific direct sequencing using PCR primers as described above.

\section{Ethical consideration}

Written informed consent for genetic investigations was obtained from all patients and family members, as well as from volunteers. The Fukuoka University Ethics Committee investigated and approved this research project (12-12 [08-77]).

Table 2 Primers for amplifying and sequencing the CACT gene

PCR

\begin{tabular}{|c|c|c|c|c|c|}
\hline product & Exon & Primer & Sequence $\left(5^{\prime}-3^{\prime}\right)$ & Size (bp) & $\operatorname{Tm}\left({ }^{\circ} \mathrm{C}\right.$ \\
\hline \multirow[t]{2}{*}{ CACT 1} & 1 & $1 \mathrm{~F}$ & GGTCGAGAACTGCAGACGGAG & 247 & 70 \\
\hline & & $1 \mathrm{R}$ & ACACATGCCCTCTTCTGCCCAG & & \\
\hline \multirow[t]{2}{*}{ САCT 2} & 2 & $2 \mathrm{~F}$ & CAGGCAGTTCTGATTCTGGT & 194 & 60 \\
\hline & & $2 \mathrm{R}$ & AACCCCGTGAATGTGTTCTG & & \\
\hline \multirow[t]{2}{*}{ САСТ 3} & 3 & $3 F$ & AAAGGTGGTGGTGTCTGTAAAC & 228 & 64 \\
\hline & & $3 R$ & GTCACGCTACCAGGCACAAC & & \\
\hline \multirow[t]{2}{*}{ САCT 4} & 4 & $4 \mathrm{~F}$ & CTCGGTGGTTAGTCACAGG & 251 & 66 \\
\hline & & $4 \mathrm{R}$ & GCCACTGCACCCAGTCCTGA & & \\
\hline \multirow[t]{2}{*}{ САCT 5} & 5 & $5 \mathrm{~F}$ & СCTGCTGGGTCTGTGACTCTGA & 224 & 70 \\
\hline & & $5 R$ & CCACTTCAGGTGACCTTCCCCA & & \\
\hline \multirow[t]{2}{*}{ САCT 6} & 6 & $6 \mathrm{~F}$ & TGGCGAAGAGTTTATGTACTTT & 233 & 60 \\
\hline & & $6 \mathrm{R}$ & CAGACATGGAGCCAAGAACA & & \\
\hline \multirow[t]{2}{*}{ САCT 7} & 7 & $7 F$ & GCCACTCTCACAGCCTTTGTCTA & 237 & 70 \\
\hline & & $7 \mathrm{R}$ & TATGAGCTTTGCACCCCAGGATTA & & \\
\hline \multirow[t]{2}{*}{ САСТ 8} & 8 & $8 \mathrm{~F}$ & GACTTAACTCGTAGTTTCTCCT & 255 & 62 \\
\hline & & $8 R$ & GGAACAAGCAAAAGTCAAACCA & & \\
\hline \multirow[t]{2}{*}{ САСТ 9} & 9 & $9 F$ & AATAGCCTATGAATAGTTATTCC & 187 & 66 \\
\hline & & $9 \mathrm{R}$ & TTAСТАСТССТTСТССТСААСGA & & \\
\hline
\end{tabular}

Abbreviation: CACT, carnitine-acylcarnitine translocase. 


\section{RESULTS}

DHPLC analysis and subsequent DNA sequencing of the CACT gene

We used DHPLC to facilitate the screening of unknown mutations in the CACT gene. PCR-amplified fragments of all nine CACT exons from a randomly chosen healthy volunteer were directly sequenced and were found to have no mutations. On using autologous DHPLC analysis, no heteroduplex pattern was found in any exon (Figure 1); therefore, this DNA fragment was used as the reference for further DHPLC studies.

We performed DHPLC analysis for nine exons of the CACT gene amplified from Case 1, Case 2, and their parents. As expected, we found heteroduplex patterns at exons 3 and 6 (namely, CACT 3 and CACT 6, respectively) in Case 1 , as well as exon 2 (CACT 2) and CACT 6 in Case 2 (Figure 2). The segregations of CACT heteroduplexes were subsequently confirmed by family studies.

Our results from direct DNA sequencing of the CACT gene showed that Case 1 had the c.576G $>\mathrm{A}$ and c.199-10t $>$ g mutations. The former mutation, which has never been described elsewhere, introduces a premature stop codon at the amino-acid residue 192, tryptophan, whereas the latter is a previously reported splicing acceptor mutation at the intron 1/exon 2 junction and results in the skipping of both exons 3 and 4 or only exon $3 .{ }^{17}$ The genetic segregation was subsequently confirmed by family studies using genomic DNA from his parents (data not shown). Based on these genetic analyses, we confirmed that Case 1 had CACT deficiency.

For Case 2, at first only genomic DNA samples from the parents were sequenced, because the only samples that were available for Case 2 were a few residual blood spots obtained for neonatal screening. We found that the father had c.106-2a $>t$ at the splice acceptor site of intron 1, which was another novel mutation identified in this study, whereas the mother had the c.576G $>$ A mutation in exon 6 , the same novel mutation shared by Case 1 . As Case 2 was found to have a normal CPT2 gene, we highly suspected that he had CACT deficiency. Next, exons 2 and 6 of Case 2 were sequenced to determine the presence of these mutations. The DHPLC and partial sequencing results described above, together with full-length exon sequencing and DHPLC of the CACT gene of his parents, confirmed the presence of the c.106-2a $>t$ mutation at the junction of intron 1 and exon 2, as well as c.576G $>\mathrm{A}$ in exon 6 . Based on these genetic analyses, Case 2 was finally diagnosed with CACT deficiency. To the best of our knowledge, the c.576G $>$ A and c.106-2a $>$ t genotypes have never been reported in the literature. It is also worth noting that c.576G $>$ A was seen in both Cases 1 and 2 .

In parallel, we investigated CACT mutations in 18 patients with non-traumatic rhabdomyolysis and 58 healthy volunteers by using DHPLC. Among the 18 patients, only 1 heteroduplex was found in exon 8 (CACT 8; Figure 2). The mutation, identified as c.804delG by DNA sequencing, resulted in a frameshift at codon 269. This patient, Case 3, was a 74-year-old Japanese man with hypertension and previously undetected rhabdomyolysis (peak serum creatine kinase level, $4832 \mathrm{IU} \mathrm{ml}^{-1}$ ) with renal injury (serum creatinine level, $2.2 \mathrm{mg} \mathrm{dl}^{-1}$ ). The CACT enzymatic activity in the fibroblasts was determined on a later day and found to be within the normal range $\left(101 \mathrm{pmol} \cdot \mathrm{min}^{-1} \cdot \mathrm{mg}^{-1}\right.$ vs the normal range, $59-308 \mathrm{pmol} \cdot \mathrm{min}^{-1}$ $\cdot \mathrm{mg}^{-1}$; data from Dr Ronald J. A. Wanders). ${ }^{18}$

Among the 58 healthy individuals, 1 heteroduplex was found at exon 5 of the CACT gene (CACT 5). This individual was a 33-year-old man, namely, Case 4. This exon 5 mutation was subsequently confirmed by DNA sequencing as c.516T $>C$, which resulted in the synonymous mutation T172T (Figure 2). This genetic alteration is the third novel CACT mutation identified in this report.

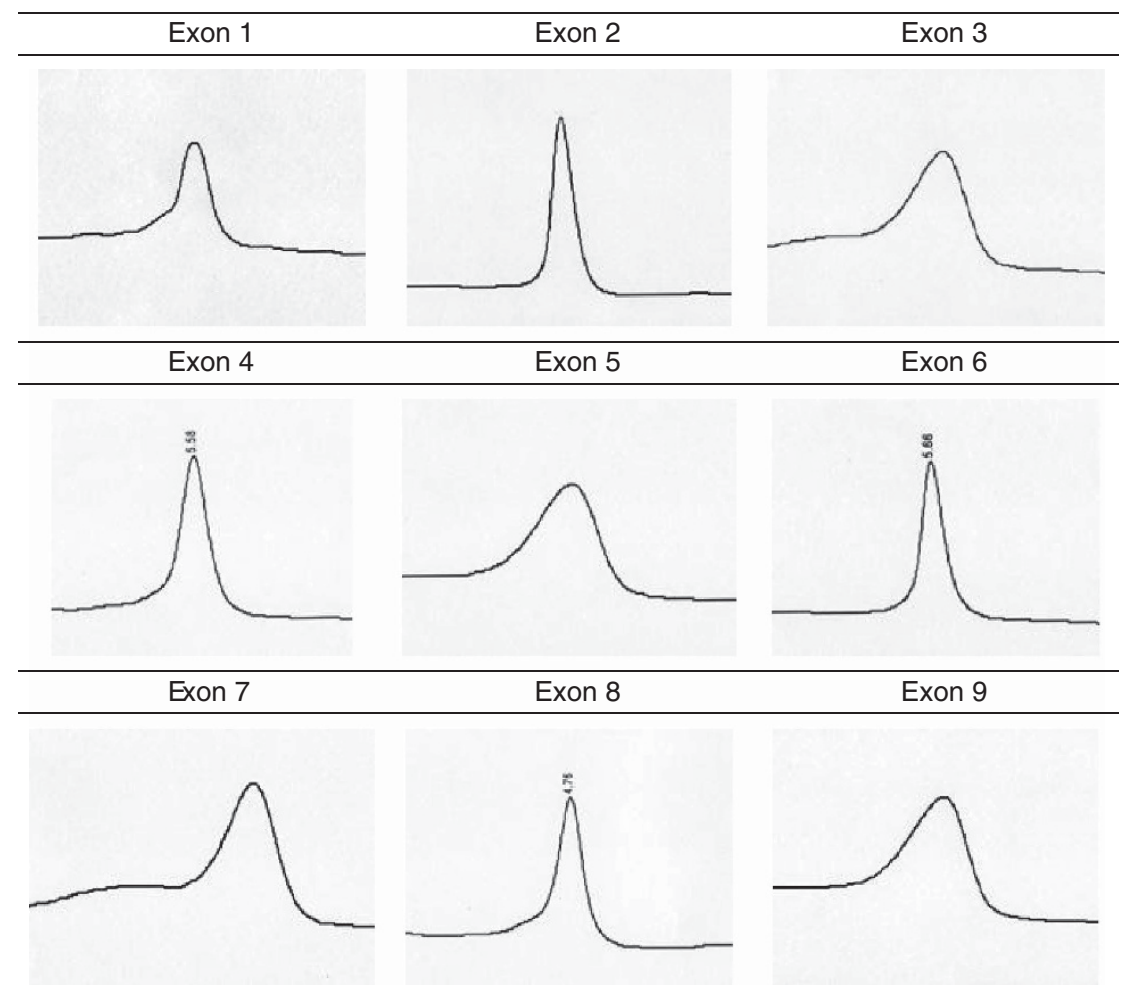

Figure 1 DHPLC patterns of homoduplexes obtained with normal CACT exons. Complete sequence-matched hybridization between two normal or autologous sequences produced a single peak for each exon. 

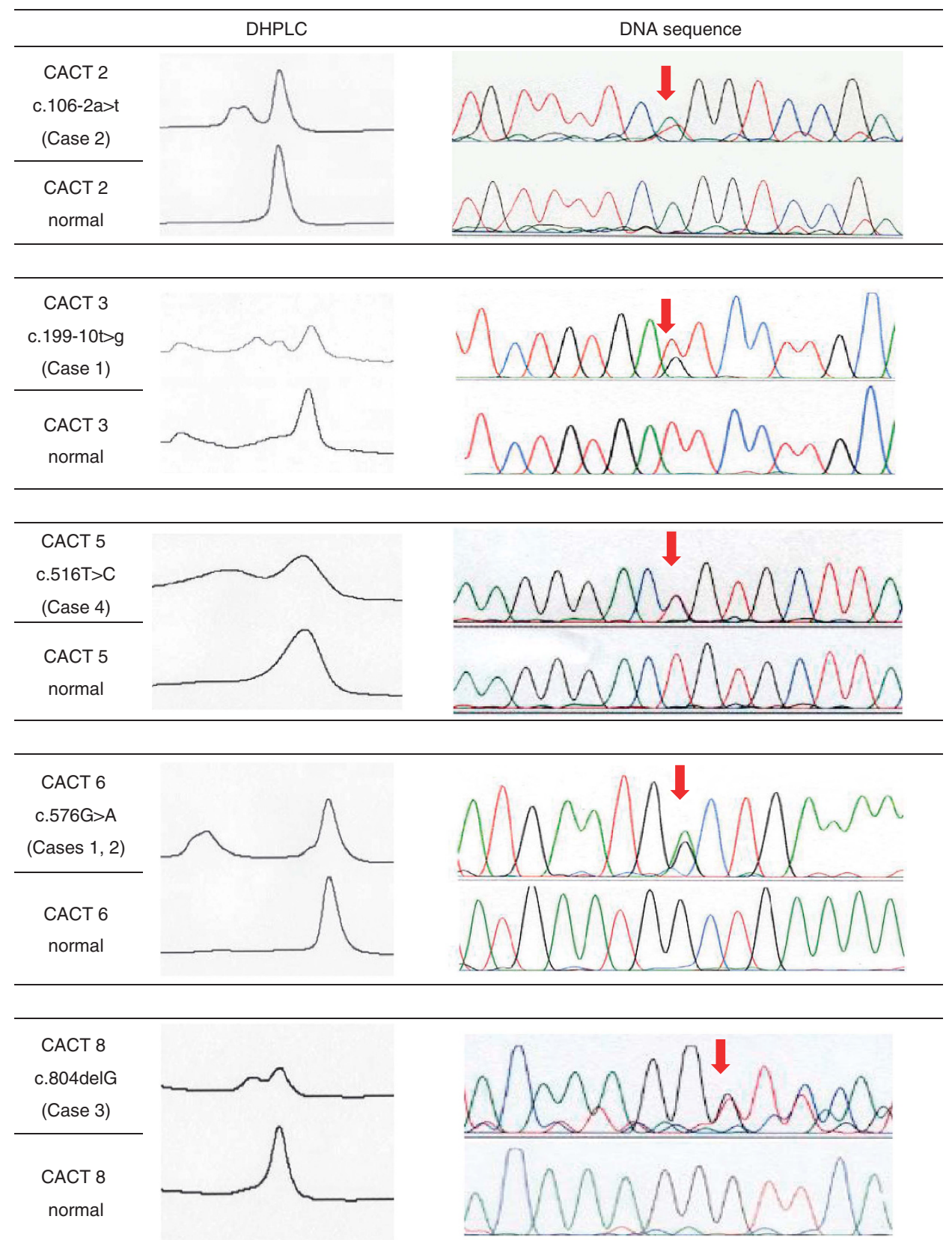

Figure 2 Aberrant DHPLC patterns of heteroduplexes and corresponding DNA sequences. Hybridization of unmatched normal and mutated sequences generated heteroduplexes, thereby resulting in aberrant peaks with different retention times. Red arrows indicate positions of mutations identified by direct DNA sequencing.

To confirm the reliability of DHPLC, 13 healthy individuals without any DHPLC heteroduplex were randomly selected, and their DNA was subjected to full-length DNA sequencing. No mutations were found in any exon of their CACT gene (data not shown).

\section{DISCUSSION}

CACT, as well as CPT2, is one of the key enzymes that participate in the intake of long-chain fatty acids into the mitochondrial matrix; therefore, defective activities of these enzymes result in impaired $\beta$-oxidation of fatty acids and lack of indispensable energy. CPT2 deficiency and CACT deficiency are known to induce a wide range of clinical manifestations, from lethal neonatal episodes of coma due to hypoketotic hypoglycemia, cardiomyopathy and cardiac arrhythmia to the adult-type of non-traumatic rhabdomyolysis. ${ }^{5,8,19}$ CPT2 deficiency consists of a lethal neonatal form, an infantile (hepatic) form and an adult-onset (muscular) form. The genetic characteristics of CPT2 deficiency have been well analyzed. We have previously described gene mutations in seven patients with definitive CPT2 deficiency, and by determining their genotypes, we found Japanese patient-specific mutations; however, we failed to find any relationship between their genotypes and clinical manifestations. ${ }^{15,20,21}$ On the other hand, the genetic characteristics of CACT deficiency have not been well described, due to the scarcity of the disease. To the best of our knowledge, only 40 patients at most have been reported 
Table 3 Mutations reported in this study

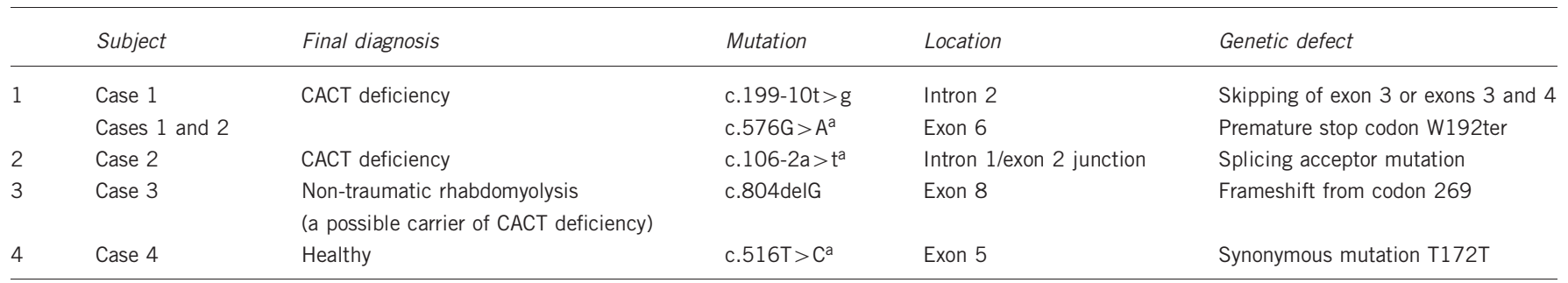

Abbreviation: CACT, carnitine-acylcarnitine translocase.

${ }^{a}$ Newly identified mutations in this study.

worldwide in the literature, and among them, the partial genotype of one Japanese patient was briefly described by a researcher from our group (TF) ${ }^{6,7,22,23}$ In many cases, CACT deficiency was seen during the neonatal period, with a few cases seen in their infantile period. The rarity of the disease may be explained in part by miscarriage and stillbirth.

Because of the resemblance of clinical features, including the acylcarnitine profiles determined by tandem mass spectrometry, between CACT deficiency and the neonatal form of CPT2 deficiency, genotyping is expected to be a pivotal tool for differentially diagnosing these two disorders. Here, we report the genotypes of two cases with CACT deficiency. Two CACT mutations, c.576G $>$ A and c.106$2 \mathrm{a}>\mathrm{t}$, have never been reported in the literature; the former mutation was detected in both the affected babies and could be Japanese patient-specific. The c.576G $>$ A mutation changes the tryptophan at the amino-acid residue 192 to a stop signal. The three-dimensional structure of rat CACT indicates that such a mutation might result in an incomplete binding-site structure for cytosomal fatty acids and therefore lead to impaired enzymatic functions. ${ }^{24}$ The importance of the splicing mutation c.106-2a $>t$ has been emphasized by Korman et al..$^{25}$ Another identified mutation, c.199-10t>g, was the commonest one among the patients reported, especially the Chinese population. ${ }^{7}$

Among the patients with non-traumatic rhabdomyolysis, we found the c.804delG mutation in the CACT gene, which has been reported to be pathogenetic in a Cape Indian individual and a Caucasian individual. ${ }^{7}$ Although heterozygous CPT2 deficiency has also been reported to cause rhabdomyolysis, ${ }^{26}$ we propose that our patient bearing this mutation was a heterozygous carrier of CACT deficiency, because the CACT enzymatic activity of his fibroblasts was maintained within the normal range. Acylcarnitine analysis was not performed for these patients; hence, we could not completely exclude the possibility that they were affected by other $\beta$-oxidation defects, such as very long chain acyl-CoA dehydrogenase (VLCAD) deficiency. However, the incidence of VLCAD deficiency is believed to be less than that of CPT2 deficiency in the Japanese population. ${ }^{27}$ c.516T $>\mathrm{C}$ was found to be a synonymous variant in the case of the healthy volunteers. Table 3 summarizes all the genetic variations found in this study.

CACT deficiency needs to be diagnosed genetically as early as possible because of the following reasons: (1) patients with the disorder die during the neonatal period, (2) tests for measurement of the enzyme activity are not necessarily available worldwide, (3) differential diagnosis between CPT2 and CACT deficiency can be achieved only by genetic methods, (4) immediate induction of adequate therapies is required, and (5) genetic analysis can distinguish patients with CACT deficiency-like diseases but without genetic mutations. $^{28,29}$ However, due to the rarity of the disease, the structure and characteristics of the CACT gene are still not well understood. Recently, DHPLC has been developed to screen mutations in these types of rare diseases, as it is particularly useful for detecting unknown polymorphisms and mutations. In this study, we demonstrate that all the PCR products producing heteroduplexes during DHPLC indeed contained mutated sequences, whereas normal sequences did not cause heteroduplex formation. These findings were further confirmed by family studies of each CACT-deficiency patient.

In conclusion, we identified five mutations in the CACT gene, three of which were responsible for CACT deficiency. We have also demonstrated the successful screening of unknown CACT gene mutations with DHPLC.

\section{ACKNOWLEDGEMENTS}

We deeply thank the two deceased babies and their parents, patients, and healthy volunteers for participating in this study. We also thank Dr Minako Sugiyama at the Department of Pediatrics, Hokkaido University, Hokkaido, Japan for letting us genotype the CACT gene of Case 1, as well as Professor Ronald J. A. Wanders at the University of Amsterdam, Amsterdam, the Netherlands for evaluating the CACT activity from the patient with nontraumatic rhabdomyolysis. This work was supported financially in part by research fund no. 086004 from the Central Research Institute of Fukuoka University, Fukuoka, Japan (HK).

1 Pande, S. V. A mitochondrial carnitine acylcarnitine translocase system. Proc. Natl Acad. Sci. USA 72, 883-887 (1975).

2 Huizing, M., lacobazzi, V., Ijlst, L., Savelkoul, P., Ruitenbeek, W., van den Heuvel, L. et al. Cloning of the human carnitine-acylcarnitine carrier CDNA and identification of the molecular defect in a patient. Am. J. Hum. Genet. 61, 1239-1245 (1997).

3 Viggiano, L., lacobazzi, V., Marzella, R., Cassano, C., Rocchi, M. \& Palmieri, F. Assignment of the carnitine/acylcarnitine translocase gene (CACT) to human chromosome band 3p21.31 by in situ hybridization. Cytogenet. Cell Genet. 79, 62-63 (1997)

4 lacobazzi, V., Naglieri, M. A., Stanley, C. A., Wanders, R. J. \& Palmieri, F. The structure and organization of the human carnitine/acylcarnitine translocase (CACT) gene. Biochem. Biophy. Res. Comm. 252, 770-774 (1998).

5 Stanley, C. A., Hale, D. E., Berry, G. T., Deleeuw, S., Boxer, J. \& Bonnefont, J. P. Brief report: a deficiency of carnitine-acylcarnitine translocase in the inner mitochondrial membrane. N. Engl. J. Med. 327, 19-23 (1992).

6 Indiveri, C., Iacobazzi, V., Tonazzi, A., Giangregorio, N., Infantino, V., Convertini, P. et al. The mitochondrial carnitine/acylcarnitine carrier: function, structure and physiopathology. Mol. Aspects Med. 32, 223-233 (2011).

7 Wang, G. L., Wang, J., Douglas, G., Browning, M., Hahn, S., Ganesh, J. et al. Expanded molecular features of carnitine acyl-carnitine translocase (CACT) deficiency by comprehensive molecular analysis. Mol. Genet. Metab. 103, 349-357 (2011).

8 Pande, S. V., Brivet, M., Slama, A., Demaugre, F., Aufrant, C. \& Saudubray, J. M. Carnitine-acylcarnitine translocase deficiency with severe hypoglycemia and auriculo ventricular block. Translocase assay in permeabilized fibroblasts. J. Clin. Invest. 91, 1247-1252 (1993).

9 Lopriore, E., Gemke, R. J., Verhoeven, N. M., Jakobs, C., Wanders, R. J., Roeleveld Versteeg, A. B. et al. Carnitine-acylcarnitine translocase deficiency: phenotype, residual enzyme activity and outcome. Eur. J. Pediatr. 160, 101-104 (2001).

10 Fingerhut, R., Röschinger, W., Muntau, A. C., Dame, T., Kreischer, J., Arnecke, R. et al. Hepatic carnitine palmitoyltransferase I deficiency: acylcarnitine profiles in blood spots are highly specific. Clin. Chem. 47, 1763-1768 (2001). 
11 Roe, D. S., Yang, B. Z., Vianey-Saban, C., Struys, E., Sweetman, L. \& Roe, C. R. Differentiation of long-chain fatty acid oxidation disorders using alternative precursors and acylcarnitine profiling in fibroblasts. Mol. Genet. Metab. 87, 40-47 (2006).

12 Yang, B. Z., Mallory, J. M., Roe, D. S., Brivet, M., Strobel, G. D., Jones, K. M. et al. Carnitine/acylcarnitine translocase deficiency (neonatal phenotype): successful prenatal and postmortem diagnosis associated with a novel mutation in a single family. Mol. Genet. Metab. 73, 64-70 (2001).

13 Rubio-Gozalbo, M. E., Vos, P., Forget, P. P., Van Der Meer, S. B., Wanders, R. J., Waterham, H. R. et al. Carnitine-acylcarnitine translocase deficiency: case report and review of the literature. Acta Paediatr. 92, 501-504 (2003).

14 Kosaki, K., Udaka, T. \& Okuyama, T. DHPLC in clinical molecular diagnostic services. Mol. Genet. Metab. 86, 117-123 (2005).

15 Kaneoka, H., Uesugi, N., Moriguchi, A., Hirose, S., Takayanagi, M., Yamaguchi, S. et al. Carnitine palmitoyltransferase II deficiency due to a novel gene variant in a patient with rhabdomyolysis and ARF. Am. J. Kidney Dis. 45, 596-602 (2005).

16 Kaneoka, H., Hsu, K. C., Takeda, Y., Sharp, G. C. \& Hoffman, R. W. Molecular genetic analysis of HLA-DR and HLA-DQ genes among anti-U1-70-kd autoantibody positive connective tissue disease patients. Arthritis. Rheum. 35, 83-94 (1992).

17 Ogawa, A., Yamamoto, S., Kanazawa, M., Takayanagi, M., Hasegawa, S. \& Kohno, Y. Identification of two novel mutations of the carnitine/acylcarnitine translocase (CACT) gene in a patient with CACT deficiency. J. Hum. Genet. 45, 52-55 (2000).

18 IJlst, L., van Roermund, C. W. T., lacobazzi, V., Oostheim, W., Ruiter, J. P. N., Williams, J. C. et al. Functional analysis of mutant human carnitine acylcarnitine translocases in yeast. Biochem. Biophys. Res. Comm. 280, 700-706 (2001).

19 Lee, R. S., Lam, C. W., Lai, C. K., Yuen, Y. P., Chan, K. Y., Shek, C. C. et al. Carnitineacylcarnitine translocase deficiency in three neonates presenting with rapid deterioration and cardiac arrest. Hong Kong Med. J. 13, 66-68 (2007).

20 Aoki, J., Yasuno, T., Sugie, H., Kido, H., Nishino, I., Shigematsu, Y. et al. A Japanese adult form of CPT II deficiency associated with a homozygous F383Y mutation. Neurology 69, 804-806 (2007).
21 Yasuno, T., Kaneoka, H., Tokuyasu, T., Aoki, J., Yoshida, S., Takayanagi, M. et al. Mutations of carnitine palmitoyltransferase II (CPT II) in Japanese patients with CPT II deficiency. Clin. Genet. 73, 496-501 (2008).

22 Wilson, C., Kerruish, N. J., Wilcken, B., Wiltshire, E., Bendikson, K. \& Webster, D. Diagnosis of disorders of intermediary metabolism in New Zealand before and after expanded newborn screening: 2004-2009. N. Z. Med. J. 125, 42-50 (2012).

23 Yamaguchi, S., Li, H., Purevsuren, J., Yamada, K., Furui, M., Takahashi, T. et al. Bezafibrate can be a new treatment option for mitochondrial fatty acid oxidation disorders: evaluation by in vitro probe acylcarnitine assay. Mol. Genet. Metab. 107, 87-91 (2012)

24 Tonazzi, A., Giangregorio, N., Indiveri, C. \& Palmieri, F. Identification by site-directed mutagenesis and chemical modification of three vicinal cysteine residues in rat mitochondrial carnitine/acylcarnitine transporter. J. Biol. Chem. 280, 19607-19612 (2005).

25 Korman, S. H., Pitt, J. J., Boneh, A., Dweikat, I., Zater, M., Meiner, V. et al. A novel SLC25A20 splicing mutation in patients of different ethnic origin with neonatally lethal carnitine-acylcarnitine translocase (CACT) deficiency. Mol. Genet. Metab. 89, 332-338 (2006).

26 Anichini, A., Fanin, M., Vianey-Saban, C., Cassandrini, D., Fiorillo, C., Bruno, C. et al. Genotype-phenotype correlations in a large series of patients with muscle type CPT II deficiency. Neurol. Res. 33, 24-32 (2011).

27 Laforet, P., Acquaviva-Bourdain, C., Rigal, O., Brivet, M., Penisson-Besnier, I., Chabrol, B. et al. Diagnostic assessment and long-term follow-up of 13 patients with very long-chain acyl-coenzyme A dehydrogenase (VLCAD) deficiency. Neuromus. Dis. 19, 324-329 (2009).

28 Iacobazzi, V., Invernizzi, F., Baratta, S., Pons, R., Chung, W., Garavaglia, B. et al. Molecular and functional analysis of SLC25A20 mutations causing carnitine-acylcarnitine translocase deficiency. Hum. Mutat. 24, 312-320 (2004).

29 Sekoguchi, E., Sato, N., Yasui, A., Fukada, S., Nimura, Y., Aburatani, H. et al. A novel mitochondrial carnitine-acylcarnitine translocase induced by partial hepatectomy and fasting. J. Biol. Chem. 278, 38796-38802 (2003). 